\title{
A randomized-controlled trial of prolotherapy injections in the treatment of plantar fasciitis
}

\author{
Ömer Ersen, ${ }^{1}$ Kenan Koca, ${ }^{1}$ Serkan Akpancar, ${ }^{1}$ Mehmet Murat Seven, ${ }^{2}$ Faruk Akyldız, ${ }^{1}$ Yavuz Ylldız, Hüseyin Özkan ${ }^{1}$ \\ 'Department of Orthopedics and Traumatology, Gulhane Training and Research Hospital, Ankara, Turkey \\ ${ }^{2}$ Department of Sports Medicine, Gulhane Training and Research Hospital, Ankara, Turkey
}

Received: October 06, 2016 Accepted: February 06, 2017 Published online: August 12, 2017

\begin{abstract}
Objectives: This study aims to evaluate the efficacy of prolotherapy injections for the treatment of plantar fasciitis.

Material and methods: Between October 2014 and October 2015, 60 patients with symptomatic chronic plantar fasciitis were randomly divided into two groups, as control $(n=31)$ and prolotherapy $(n=29)$ groups. In the prolotherapy group, ultrasound-guided prolotherapy injections into the plantar fascia up to five different points were performed three times every 21 days. In the control group, the patients received instructions for plantar fascia and Achilles tendon stretching exercises three times a week for three months. Additionally, all patients were given heel lifts and instructed to refrain from heavy loading activity. The patients were evaluated via the Visual Analog Scale (VAS), Food and Ankle Outcome Score (FAOS), and Foot Function Index (FFI) at baseline and at 21, 42, 90, and 360 days during follow-up.

Results: A total of 50 patients completed follow-up (26 patients in the prolotherapy group and 24 patients in the control group). The VAS, FAOS, and FFI scores were significantly improved in both groups $(\mathrm{p}<0.001)$. There were no statistically significant differences in the pain and functional scores at 21 days of treatment between the groups. The VAS and FAOS scores were higher in the prolotherapy group than the control group at 42, 90, and 360 days of treatment. The FFI scores were also higher in the prolotherapy group than the controls at 42 and 90 days of treatment; however, both groups had similar scores at 360 days.
\end{abstract}

Conclusion: Our study results suggest that prolotherapy is an effective auxiliary method for treating chronic plantar fasciitis.

Keywords: Plantar fascia-specific stretching exercises; plantar fasciitis; prolotherapy injections.

Plantar fasciitis is a common source of heel pain, commonly afflicting women rather than men, and is one of the most common causes of disability in patients aged 40 and 60 years. ${ }^{[1,2]}$ Several treatment methods are available. Classical methods include shoe modification, the use of prefabricated and custom orthotics, dorsiflexion night splints, non-steroidal anti-inflammatory drugs, and steroid injections. New methods include the use of platelet-rich plasma (PRP), botulinum toxin type A, dry needling and stretching exercises for the gastrocnemius and soleus muscles and the plantar fascia in addition to the application of extracorporeal shock waves. ${ }^{[3-5]}$ Symptoms often reduce within 10 months of the use of the classical modalities, but persist or become exacerbated in approximately $10 \%$ of cases. ${ }^{[6]}$ Therefore, the need for more effective treatment modalities for this patient population still remains.

Prolotherapy has been successfully used in the treatment of tendinopathy and other musculoskeletal conditions in recent years. ${ }^{[7-9]}$ Prolotherapy injections are prepared with hypertonic dextrose in a distinct concentration which causes the osmotic rupture of local cells. ${ }^{[9]}$ This increases glucose in the extracellular matrix, thereby, increasing the growth factors and subsequent healing. Deoxyribonucleic acid (DNA)-encoding growth factors have been also shown to contribute to an increase in the hypertonic environment. ${ }^{[10]}$ Recently, prolotherapy has been successfully used in sprained and degenerated ligaments, and damaged, dense connective tissue

Corresponding author: Kenan Koca, MD. Gülhane Ĕ̌itim Araştırma Hastanesi Ortopedi ve Travmatoloji Kliniği, 06010 Etlik, Ankara, Turkey. e-mail: drkenankoca@gmail.com 
structures, including tendons and entheses. ${ }^{[11,12]}$ Easy application, high success rate, low associated complication risks, and short rehabilitation process are the reasons why prolotherapy is the preferred treatment for these conditions. It also facilitates healing of the ligaments and tendon structures after function has been restored.$^{[9,12-14]}$ In addition, serious side-effects or adverse events were not reported in relation to prolotherapy, when it was used for the previously mentioned indications. ${ }^{[9,15]}$

Despite proliferation of the aforementioned treatment methods for plantar fasciitis, there is a limited number of evidence on prolotherapy. ${ }^{[14]}$ Therefore, in the present study, we aimed to evaluate the efficacy and safety of prolotherapy injections for the treatment of plantar fasciitis.

\section{MATERIALS AND METHODS}

\section{Study design and follow-up}

This prospective, randomized-controlled study was approved by Gülhane Military Medical Academy Ethical Committee. All patients were fully informed and a written informed consent was obtained. The study was conducted in accordance with the principles of the Declaration of Helsinki.

A total of 60 patients diagnosed with plantar fasciitis were included in the study between October 2014 and October 2015. All patients were randomly divided into two groups by computer (prolotherapy $[\mathrm{n}=29]$ and control [ $\mathrm{n}=31]$ groups). Diagnosis was based on the identification of symptoms and physical examination findings. A lateral radiograph of the ankle was taken to exclude epin calcanei. Patients with tarsal tunnel syndrome and epin calcanei were excluded from the study.

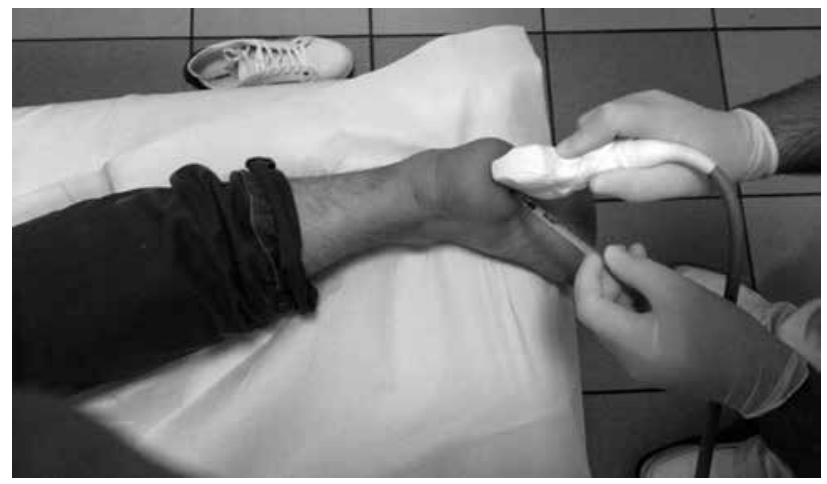

Figure 1. Ultrasound guided injection.

\section{Study procedures}

Ultrasound-guided prolotherapy injections with a 27-gauge needle $(3.6 \mathrm{~mL}$ dextrose [15\% solution] and $0.4 \mathrm{~mL}$ lidocaine) were administered in up to five different points in the plantar fascia under aseptic conditions in the prolotherapy group. The medialoblique approach was used for the injections. ${ }^{[16]}$ An ultrasound probe was placed on the medial calcaneal tubercle. The needle was inserted from the medial side of the heel, perpendicular to the long axis of the ultrasound transducer, and advanced under continuous ultrasound guidance into the proximal plantar fascia. Sterile transmission gel and transducer covers were employed throughout the injection procedure (Figure 1). The prolotherapy injections were administered three times in every patient every three weeks. The use of anti-inflammatory agents was not permitted.

The control group performed plantar fascia and Achilles tendon stretching exercises three times a week for three months, as described by Digiovanni et al. ${ }^{[17]}$
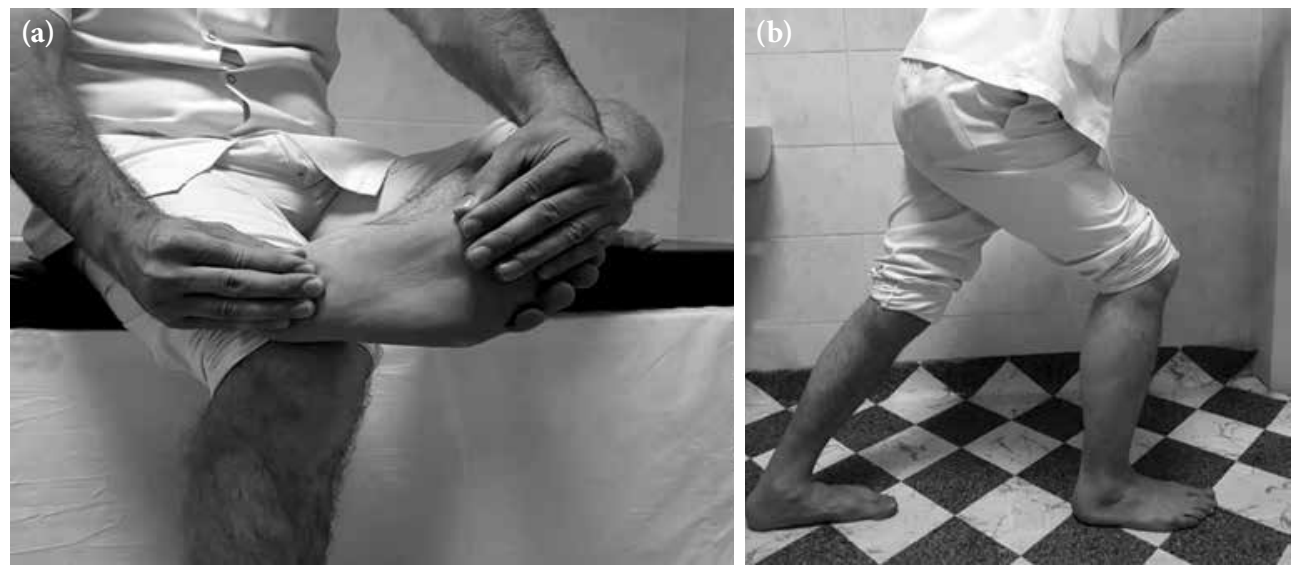

Figure 2. (a) Plantar fascia. (b) Achilles tendon stretching exercises. 


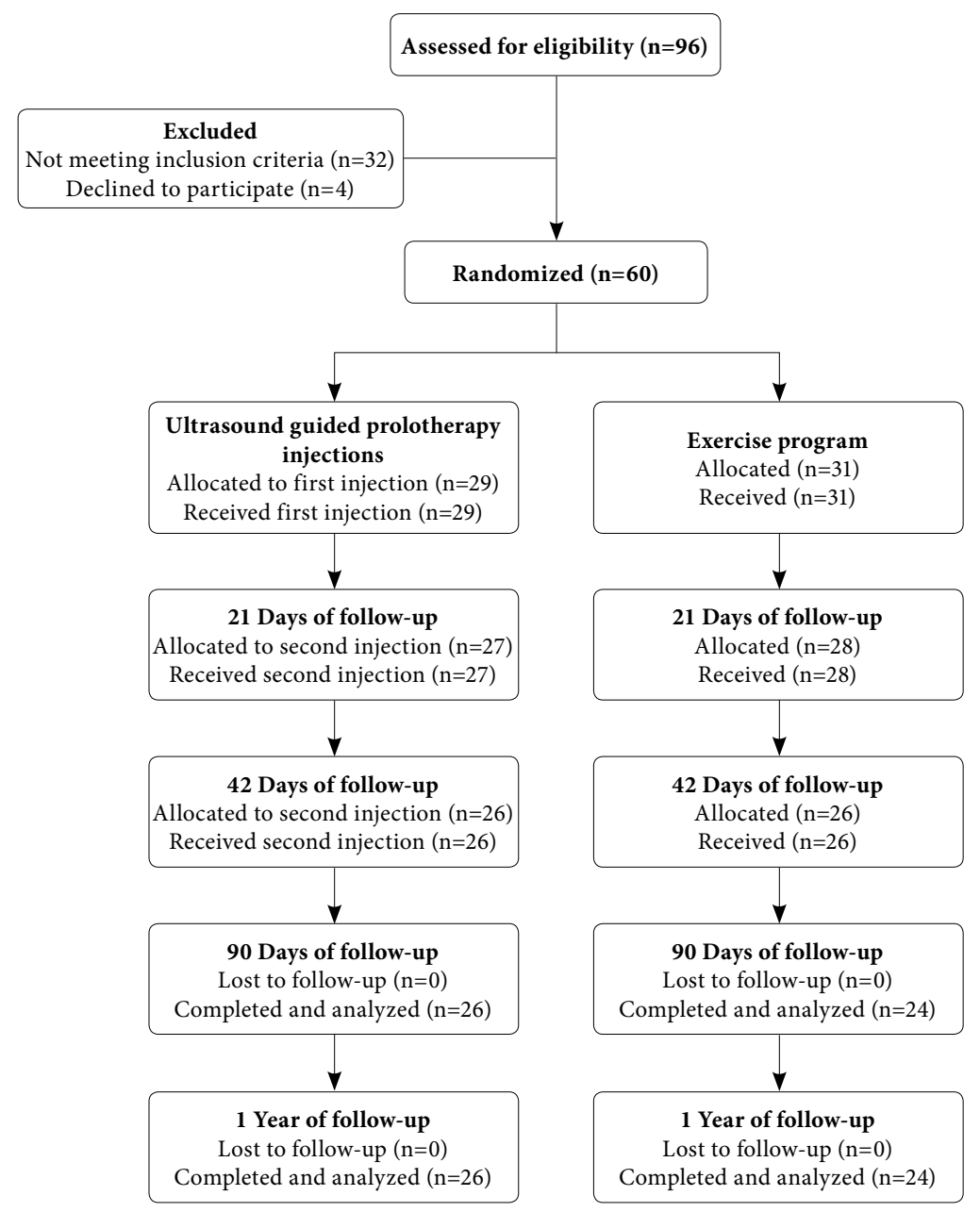

Figure 3. Flowchart of subjects in the study.

A physical therapist with a three-year experience provided instructions on the manual exercises. The patients were also advised to perform a home-based exercise program with the same exercise protocol on their own three times a day for the other days (Figure 2).

Additionally, all patients were given heel lifts and instructed to refrain from heavy loading activities.

Table 1. Baseline demographic characteristics of the groups

\begin{tabular}{|c|c|c|c|c|c|}
\hline & \multicolumn{2}{|c|}{ Prolotherapy group $(n=26)$} & \multicolumn{2}{|c|}{ Control group $(n=24)$} & \multirow[b]{2}{*}{$p$} \\
\hline & $\mathrm{n}$ & Mean \pm SD & $\mathrm{n}$ & Mean \pm SD & \\
\hline Age (years) & & $45.1 \pm 6.7$ & & $46.3 \pm 7.6$ & 0.539 \\
\hline Sex & & & & & 0.623 \\
\hline Female & 21 & & 19 & & \\
\hline Male & 5 & & 6 & & \\
\hline Time of symptoms (month) & & $32.8 \pm 23.9$ & & $34.3 \pm 23.3$ & 0.825 \\
\hline Side & & & & & 0.616 \\
\hline Right & 18 & & 15 & & \\
\hline Left & 8 & & 9 & & \\
\hline Visual Analog Scale (Baseline) & & $6.9 \pm 1.5$ & & $6.7 \pm 1.44$ & 0.600 \\
\hline AOFAS (Baseline) & & $55.0 \pm 15.5$ & & $57.4 \pm 14.4$ & 0.683 \\
\hline Foot Function Index (Baseline) & & $57.7 \pm 13.6$ & & $56.9 \pm 12.7$ & 0.834 \\
\hline
\end{tabular}

SD: Standard deviation; AOFAS: American Orthopedic Foot and Ankle Society Clinical Rating System. 


\section{Patient evaluation}

The patients were evaluated at baseline and, then, at $21,42,90$, and 360 days by one of the co-authors who was blinded to the type of treatment received. Pain intensity was recorded using a 10-point Visual Analog Scale (VAS) with " 0 " being indicative of no pain and "10" severe pain. The clinical outcomes were rated as "excellent", "good", "fair" or "poor":

- An "excellent" result was defined as the absence of heel pain arising from daily living activities, including sport.

- A "good" result was defined as $\leq 50 \%$ of the original heel pain arising from certain activities, including sport.

- A "fair" result was defined as 50 to $75 \%$ of the original heel pain arising from certain activities.

- A "poor" result was defined as $\geq 75 \%$ of the original heel pain.

A specific evaluation method, that is both safe and efficacious in patients with plantar fasciitis, has not been identified, yet. Therefore, the Food and Ankle Outcome Score (FAOS) of the American Orthopedic Foot and Ankle Society Clinical Rating System (AOFAS) comprising subjective and objective components was utilized. ${ }^{[18]}$ The Foot Function Index (FFI) was also used to evaluate foot pathologies in terms of function, pain, disability, and activity restriction. ${ }^{[19]}$

\section{Statistical analysis}

Statistical analysis was performed using the IBM SPSS for Windows version 22.0 (IBM Corp., Armonk, NY, USA). Descriptive data were presented in number, percentage, and mean \pm standard deviation (SD). The congruity of the normally distributed continuous variables was evaluated using the KolmogorovSmirnov test. The chi-square $\left(\chi^{2}\right)$ test was used to assess the relationship between two categorical variables. The Student's t-test and Mann-Whitney $\mathrm{U}$ test were used for the clinical evaluation of intergroup comparisons. The Friedman test was used for intra-group analysis, and Bonferroni correction for post-hoc tests. A $p$-value of $<0.05$ was considered statistically significant.

\section{RESULTS}

Of 60 patients, 50 [prolotherapy group [n=26], control group [ $\mathrm{n}=24]$ ) completed the study protocol. Seven participants in the control group and three participants in the prolotherapy group did not attend

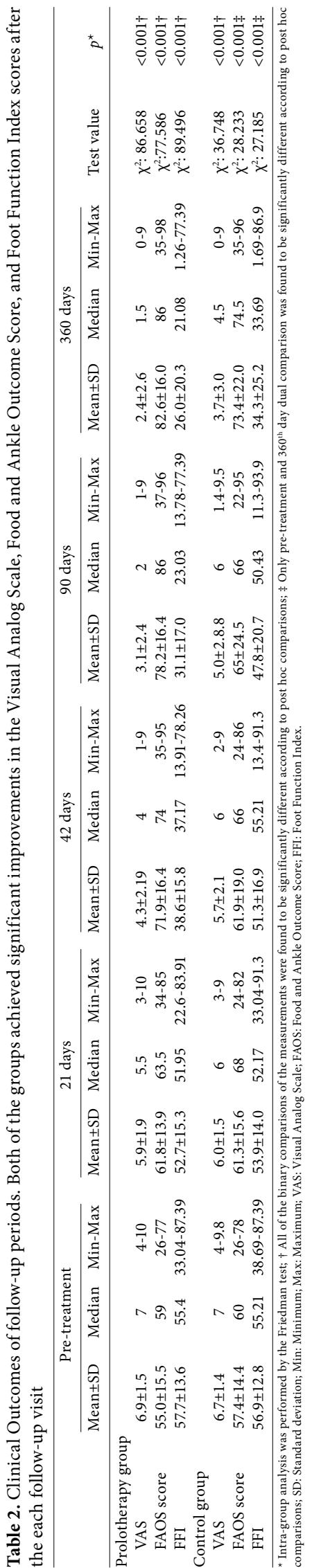


Table 3. Clinical outcomes reported at the follow-up meetings

\begin{tabular}{|c|c|c|c|c|c|c|c|c|}
\hline \multirow[t]{2}{*}{ Score } & \multicolumn{3}{|c|}{ Prolotherapy improvement ${ }^{*}$} & \multicolumn{3}{|c|}{ Control improvement ${ }^{*}$} & \multirow[b]{2}{*}{ Test value } & \multirow[b]{2}{*}{$p$} \\
\hline & Mean \pm SD & Median $\dagger$ & $\operatorname{Min-Max\dagger }$ & Mean \pm SD & Median $\dagger$ & $\operatorname{Min}-\operatorname{Max} \dagger$ & & \\
\hline VAS (0-21 days) & $-1.0 \pm 1.2$ & -1.0 & -4.0 to 1.0 & $-0.7 \pm 0.9$ & -1.0 & -2.1 to 1.0 & $z=-0.996$ & 0.319 \\
\hline VAS (0-42 days) & $-2.6 \pm 1.2$ & & & $-1.0 \pm 1.7$ & & & $\mathrm{t}=3.700$ & 0.001 \\
\hline VAS (0-90 days) & $-3.8 \pm 1.8$ & & & $-1.7 \pm 2.5$ & & & $\mathrm{t}=3.255$ & 0.002 \\
\hline VAS (0-360 days) & $-4.5 \pm 2.4$ & -4.5 & -8.5 to -0.8 & $-3 \pm 2.6$ & -2.0 & -7.5 to 0 & $\mathrm{z}=-2.033$ & 0.042 \\
\hline AOFAS (0-21 days) & $6.8 \pm 3.8$ & & & $3.8 \pm 9.2$ & & & $\mathrm{t}=-1.116$ & 0.270 \\
\hline AOFAS (0-42 days) & $16.9 \pm 4.5$ & & & $4.5 \pm 14.3$ & & & $\mathrm{t}=-3.512$ & 0.001 \\
\hline AOFAS (0-90 days) & $23.2 \pm 7.6$ & & & $7.6 \pm 19$ & & & $t=-3.367$ & 0.002 \\
\hline AOFAS (0-360 days) & $27.6 \pm 16$ & & & $16 \pm 16.4$ & & & $\mathrm{t}=-2.358$ & 0.023 \\
\hline FFI (0-21 days) & $-4.9 \pm 6.3$ & & & $-2.9 \pm 5.2$ & & & $\mathrm{t}=1.203$ & 0.235 \\
\hline FFI (0-42 days) & $-19 \pm 9.7$ & -18.91 & -40.44 to -0.43 & $-5.5 \pm 11.1$ & -3.7 & -42.25 to 10.44 & $z=-4.166$ & $<0.001$ \\
\hline FFI (0-90 days) & $-26.6 \pm 13.5$ & -27.62 & -61.31 to -1.74 & $-9.1 \pm 16.4$ & -3.92 & -44.35 to 10.0 & $z=-3.622$ & $<0.001$ \\
\hline FFI (0-360 days) & $-31.7 \pm 18$ & & & $-22.6 \pm 21.8$ & & & $\mathrm{t}=1.613$ & 0.113 \\
\hline
\end{tabular}

* The change in the outcome scores was used in between group comparison; † The variable doesn't have normal distribution; SD: Standard deviation; Min: Minimum; Max: Maximum; VAS: Visual Analog Scale; AOFAS: American Orthopedic Foot and Ankle Society Clinical Rating System; FFI: Foot Function Index.

to follow-up visits and were excluded (Figure 3). Both groups had similar demographic characteristics. The demographic characteristics of the two groups are shown in Table 1.

A significant improvement in the VAS, FAOS, and FFI scores was achieved in both groups during follow-up compared to baseline values $(\mathrm{p}<0.001)$ (Table 2). Twenty patients (77\%) in the prolotherapy group reported "good" to "excellent" outcomes, and six (27\%) "fair" and "poor" outcomes. Four patients $(16.6 \%)$ in the control group reported "good" outcomes, and 20 (83.3\%) "fair" and "poor" outcomes.

Inter-group comparisons were based on the change in the outcome scores compared to baseline. Accordingly, the VAS and FAOS scores of the prolotherapy group at 42,90 , and 360 days were significantly higher compared to the control group. In addition, the FFI scores at 42 and 90 days were significantly better in the same group compared to the control group. However, there was no statistically significant difference in the outcomes at 360 days between the groups (Table 3).

None of the patients in the groups experienced complications. Only three of them reported severe pain one to two days after injection in the prolotherapy group, which reduced after two days of non-weight bearing.

\section{DISCUSSION}

Plantar fasciitis is one of the most common causes of foot disability in adults. Although most symptoms are managed using conservative methods, in about $10 \%$ of cases persistent symptoms can cause disability to continue
In recent years, injection-based complementary procedures have been studied in the treatment of chronic plantar fasciitis. A local steroid injection into the plantar fascia is the most commonly used treatment option, and has the potential to decrease inflammation and alleviate pain. ${ }^{[8,14,20]}$ However, short-term benefits have been demonstrated with the use of corticosteroid injections, which are also associated with numerous complications, such as plantar fascia rupture, fat pad atrophy, lateral plantar nerve injury, and calcaneal osteomyelitis. ${ }^{[20,21]}$ Platelet-rich plasma is another popular treatment option, and has proven benefits of tissue healing, pain relief and an improvement in the functional status, as well as patient's satisfaction. ${ }^{[22]}$ However, PRP preparation is not without difficulties, as it involves an invasive procedure (i.e., blood drawing) and the lack of an optimized standardized preparation protocol. Currently, considerable researches have been conducted in which PRP injections for the treatment of plantar fasciitis have been studied. However, only a few small-scale randomized-controlled studies with relatively short follow-up periods have been carried out. ${ }^{[20,23]}$ The effect of a prolotherapy injection is superior to that of a corticosteroid injection, as it provides tissue healing and renewal for a considerable duration. ${ }^{[7-11]}$ It is considered to be a viable alternative to PRP with a similar healing effect on the tissue. However, it is superior to PRP in that it is simple to prepare, non-invasive, and more cost-effective. ${ }^{[7-11,24]}$

The effectiveness of prolotherapy injections in patients with chronic plantar fasciitis has been reported in the literature. Ryan et al. ${ }^{[25]}$ first used prolotherapy injections to treat 20 patients. Compared to the pre-injection values, a significant decrease in 
the VAS scores was obtained, and "good" to "excellent" results were reported by 16 patients $(80 \%)$. However, there was no control group in their study, and the patients were evaluated using VAS scores alone.

The recent study is only the second in which the effect of prolotherapy on the treatment of chronic plantar fasciitis has been investigated and, to the best of our knowledge, it is the first in which a prospective, randomized-controlled design has been employed. In the aforementioned study, the patients were evaluated using two functional scores. A significant improvement was reflected by the VAS, FAOS, and FFI scores at 42 and 90 days of follow-up, compared to the control group. "Good" and "Excellent" results were achieved in 20 of the 26 patients (77\%) in the prolotherapy group, and in four patients (17\%) in the control group. The results of this study are consistent with the findings of the previous one.

Palpation- and ultrasound-guided prolotherapy injections can be performed to treat plantar fasciitis. Chen et al. ${ }^{[26]}$ compared ultrasound- and palpationguided prolotherapy injections in patients with unilateral plantar fasciitis, and reported significantly better therapeutic outcomes with the ultrasoundguided technique. The authors also reported that precise injections into the target point in the plantar fascia increased its effectiveness and duration of action. Of note, higher recurrence rates are experienced with palpation-guided injections, and they can further deteriorate the atrophied pad. ${ }^{[25,26]}$ Therefore, in the present study, we used ultrasound-guided injections through the adoption of a medial-oblique approach to administer the injections into the target point in the plantar fascia to increase their efficacy and duration of action.

Prolotherapy is an invasive treatment method, and three injection sessions seem to be excessive and costly. At least three injection sessions were performed in previous studies in which the efficacy of prolotherapy in the treatment of various musculoskeletal conditions was investigated. Also, it was reported in some of them that the most effective benefits could be gained with repeated injections. ${ }^{[8,12,27,28]} \mathrm{We}$, therefore, preferred the use of three prolotherapy injection sessions in our study to provide maximum pain relief to, and improved function in, patients with chronic plantar fasciitis. The patient's satisfaction and VAS and FAOS scores significantly increased after each injection session.

Physiotherapy was selected for the comparative purposes with the control group, as its effectiveness has been proven in the management of chronic plantar fasciitis. ${ }^{[16]}$ Celik et al. ${ }^{[29]}$ compared the efficacy of stretching exercises with steroid injections (the most preferred method) in the treatment of plantar fasciitis, and reported that, in the long-term, effectiveness in the exercise group was significantly higher compared to the corticosteroid group. Two physiotherapy methods are described in the literature, namely calf stretching and plantar fascia-specific stretching, in the treatment of chronic plantar fasciitis. ${ }^{[30-32]}$ Strong evidence has been provided in a recent systematic review that both methods decrease pain and improve function in patients with this condition. ${ }^{[30-32]} \mathrm{A}$ significant improvement following stretching exercises was noted in our patients at all of the followup periods, similar to that reported in previous studies. However, the success of the prolotherapy injections was significantly higher compared to the physiotherapy group in terms of VAS and FAOS scores in the long-term.

Physiotherapy is one of the most non-invasive methods, and has been proven to be effective in treating plantar fasciitis. ${ }^{[16,28]}$ However, participants need to exercise under the supervision of a physiotherapist two to three times a week. The duration of the interventions should range from 6 to 12 weeks. This imposes a substantial economic burden to individuals, and health and social care systems. Most of the costs relate to lost productivity and substantial impairment of the quality of life. Prolotherapy injections can be performed in the outpatient setting. Every injection session lasts for 15 to $20 \mathrm{~min}$. Three injection sessions may be sufficient with an interval of 21 days.

On the other hand, the main limitations of this study were small sample size, the lack of a control group without plantar fasciitis, and the fact that a saline injection procedure was used in the control group. Therefore, further, large-scale studies are required to examine the effect of prolotherapy injections on the treatment of plantar fasciitis.

In conclusion, based on the improvements in our study, these results suggest that prolotherapy is an effective auxiliary method and can be used in the treatment of chronic plantar fasciitis.

\section{Declaration of conflicting interests}

The authors declared no conflicts of interest with respect to the authorship and/or publication of this article.

\section{Funding}

The authors received no financial support for the research and/or authorship of this article. 


\section{REFERENCES}

1. Riddle DL, Pulisic M, Sparrow K. Impact of demographic and impairment-related variables on disability associated with plantar fasciitis. Foot Ankle Int 2004;25:311-7.

2. Tu P, Bytomski JR. Diagnosis of heel pain. Am Fam Physician 2011;84:909-16.

3. Powell M, Post WR, Keener J, Wearden S. Effective treatment of chronic plantar fasciitis with dorsiflexion night splints: a crossover prospective randomized outcome study. Foot Ankle Int 1998;19:10-8.

4. Placzek R, Deuretzbacher G, Meiss AL. Treatment of chronic plantar fasciitis with Botulinum toxin A: preliminary clinical results. Clin J Pain 2006;22:190-2.

5. Eftekhar-Sadat B, Babaei-Ghazani A, Zeinolabedinzadeh V. WITHDRAWN: Evaluation of dry needling in patients with chronic heel pain due to plantar fasciitis. Foot (Edinb) 2012 Nov 29.

6. Davis PF, Severud E, Baxter DE. Painful heel syndrome: results of nonoperative treatment. Foot Ankle Int 1994;15:531-5.

7. Ekinci S, Tatar O, Akpancar S, Turgut H, Seven MM. A New Treatment Option in Osteoarthritis: Prolotherapy Injections. J Arthritis 2016;5:197.

8. Seven MM, Koca K, Akpancar S, Turkkan S, Uysal B, Yildiz Y, Ozkan H, Korkmaz A. Prolotherapy Injections in the Treatment of Overuse Injuries. BMMR. Online First: 15 May, 2016.

9. Rabago D, Slattengren A, Zgierska A. Prolotherapy in primary care practice. Prim Care 2010;37:65-80.

10. Krump E, Nikitas K, Grinstein S. Induction of tyrosine phosphorylation and $\mathrm{Na}+\mathrm{H}+$ exchanger activation during shrinkage of human neutrophils. J Biol Chem 1997;272:17303-11.

11. Taylor ME. Prolotherapy for peripheral joints. Aust Musculoskelet Med 2004;9:38-41.

12. Seven MM, Koca K, Akpancar S, Turkkan S, Uysal B, Yildiz $\mathrm{Y}$, et al. Promising Results of Prolotherapy in an Elderly Male with Bilateral Partial Rotator Cuff Lesions. BMMR. [Online First: 23 May, 2016].

13. Hackett GS, Hemwall GA, Montgomery GA. Ligament and Tendon Relaxation Treated by Prolotherapy. 5th ed. Oak Park, IL: Institute in Basic Life Principles; 1991.

14. Akpancar S, Seven MM, Tuzun HY, Gurer L, Ekinci S. Current Concepts of Prolotherapy in Orthopedic Surgery Arch Trauma Res. Inpress (In-press):e40447.

15. Sanderson LM, Bryant A. Effectiveness and safety of prolotherapy injections for management of lower limb tendinopathy and fasciopathy: a systematic review. J Foot Ankle Res 2015;8:60.

16. Koski JM. Ultrasound guided injections in rheumatology. J Rheumatol 2000;27:2131-8.

17. Digiovanni BF, Nawoczenski DA, Malay DP, Graci PA, Williams TT, Wilding GE, et al. Plantar fascia-specific stretching exercise improves outcomes in patients with chronic plantar fasciitis. A prospective clinical trial with twoyear follow-up. J Bone Joint Surg [Am] 2006;88:1775-81.
18. Kitaoka HB, Alexander IJ, Adelaar RS, Nunley JA, Myerson MS, Sanders M. Clinical rating systems for the anklehindfoot, midfoot, hallux, and lesser toes. Foot Ankle Int 1994;15:349-53.

19. Budiman-Mak E, Conrad KJ, Roach KE. The Foot Function Index: a measure of foot pain and disability. J Clin Epidemiol 1991;44:561-70.

20. Acevedo JI, Beskin JL. Complications of plantar fascia rupture associated with corticosteroid injection. Foot Ankle Int 1998;19:91-7.

21. Crawford F, Thomson CE. Interventions for treating plantar heel pain. The Foot 2001;11:228-50.

22. Martinelli N, Marinozzi A, Carnì S, Trovato U, Bianchi A, Denaro V. Platelet-rich plasma injections for chronic plantar fasciitis. Int Orthop 2013;37:839-42.

23. Say F, Gürler D, İnkaya E, Bülbül M. Comparison of plateletrich plasma and steroid injection in the treatment of plantar fasciitis. Acta Orthop Traumatol Turc 2014;48:667-72.

24. Lana JFSD, Santana MHA, Belangero WD, Luzo ACM. Regenerative Medicine: Sports Medicine, Orthopedic, and Recovery of Musculoskeletal Injuries (Lecture Notes in Bioengineering). 2014

25. Ryan MB, Wong AD, Gillies JH, Wong J, Taunton JE. Sonographically guided intratendinous injections of hyperosmolar dextrose/lidocaine: a pilot study for the treatment of chronic plantar fasciitis. Br J Sports Med 2009;43:303-6.

26. Chen CM, Chen JS, Tsai WC, Hsu HC, Chen KH, Lin CH. Effectiveness of device-assisted ultrasound-guided steroid injection for treating plantar fasciitis. Am J Phys Med Rehabil 2013;92:597-605.

27. Scarpone M, Rabago DP, Zgierska A, Arbogast G, Snell E. The efficacy of prolotherapy for lateral epicondylosis: a pilot study. Clin J Sport Med 2008;18:248-54.

28. Khan SA, Kumar A, Varshney MK, Trikha V, Yadav CS. Dextrose prolotherapy for recalcitrant coccygodynia. J Orthop Surg (Hong Kong) 2008;16:27-9.

29. Celik D, Kuş G, Sırma SÖ. Joint Mobilization and Stretching Exercise vs Steroid Injection in the Treatment of Plantar Fasciitis: A Randomized Controlled Study. Foot Ankle Int 2016;37:150-6.

30. McPoil TG, Martin RL, Cornwall MW, Wukich DK, Irrgang JJ, Godges JJ. Heel pain--plantar fasciitis: clinical practice guildelines linked to the international classification of function, disability, and health from the orthopaedic section of the American Physical Therapy Association. J Orthop Sports Phys Ther 2008;38:A1-A18.

31. Landorf KB, Menz HB. Plantar heel pain and fasciitis. BMJ Clin Evid 2008;2008. pii: 1111.

32. Sweeting D, Parish B, Hooper L, Chester R. The effectiveness of manual stretching in the treatment of plantar heel pain: a systematic review. J Foot Ankle Res 2011;4:19. 